\title{
NANO-MICRO LETTERS
}

\section{Optical properties of $\mathrm{ZnO}$ and $\mathrm{Mn}$-doped $\mathrm{ZnO}$ nano- crystals by vapor phase transport processes}

\author{
Z. Wang ${ }^{1,2}$, X. Y. Ma ${ }^{1, *}$, J. W. Song ${ }^{1}$ and J. H. Yao ${ }^{2}$
}

In this paper we investigated the optical properties of $\mathrm{ZnO}$ and Mn doped $\mathrm{ZnO}$ nanocrystals that were fabricated by a vapor phase transport growth process, using zinc acetate dihydrate with or without $\mathrm{Mn}$ in a constant $\mathrm{O}_{2} / \mathrm{Ar}$ mixture gas flowing through the furnace at $400 \sim 600{ }^{\circ} \mathrm{C}$, respectively. The as grown $\mathrm{ZnO}$ nanocrystals are homogeneous with a mean size of $19 \mathrm{~nm}$ observed by scanning electron microscope (SEM). The optical characteristics were analyzed by absorption spectra and photoluminescence (PL) spectra at room-temperature. For ZnO nanocrystals, a strong and predominant UV emission peaked at $377 \mathrm{~nm}$ was found in the PL spectra. For Mn doped $\mathrm{ZnO}$ nanocrystals, in addition to the strong UV emission, a strong blue emission peaked at $435 \mathrm{~nm}$ was observed as well. By doping Mn ions, the major UV emission shifts from $377 \mathrm{~nm}$ to $408 \mathrm{~nm}$, showing that Mn ions were not only incorporated into $\mathrm{ZnO}$ Nes, but also introduced an impurity level in the bandgap. Moreover, with the concentration of Mn increasing, the relative intensities of the two emissions change largely, and the photoluminescence mechanism of them is discussed.

Keywords: $\mathrm{ZnO}$ and $\mathrm{Mn}$-doped $\mathrm{ZnO}$ nanocrystals; Optical properties; Vapor phase transport growth

Citation: Z. Wang, X. Y. Ma, J. W. Song and J. H. Yao, “Optical properties of ZnO and Mn-doped ZnO nanocrystals fabrication by vapor phase transport processes", Nano-Micro Lett. 1, 45-48 (2009). doi: 10.5101/nml.v1i1.p45-48

Zinc oxide $(\mathrm{ZnO})$, a promising II-IV group semiconductor material with hexagonal wurtzite structure, has been widely applied in various fields [1] such as transducers, transparent conduction electrode, solar sells, and wide ultraviolet (UV) optoelectronic devices [2], due to its direct band gap of $3.37 \mathrm{eV}$ at room temperature and a large exciton binding energy of 60 $\mathrm{meV}$. To realize the light-emitting devices, an important issue is the fabrication of p-type conduction $\mathrm{ZnO}$ with a high concentration of hole and a low resistance. However, it is difficult to achieve low resistivity p-type $\mathrm{ZnO}$ film because of problems such as self-compensation, deep acceptor level, and low solubility of the acceptor dopants [3].

Recently, studies present that the doping of transition metal element $\mathrm{Mn}$ into $\mathrm{ZnO}$ offers a feasible mean of realizing p-type $\mathrm{ZnO}$. Mn doped $\mathrm{ZnO}$ has also the potential to be a multifunctional material with coexisting magnetic, semi-conducting and optical properties [4], which makes a fine way to tune the bandgap in making UV detector and light emitters. Moreover, it generates a new kind of $\mathrm{ZnO}$ materialdiluted magnetic semiconductor (DMS). Dietl et. Al [5,6] predicted that the transition temperature $\mathrm{T}_{\mathrm{c}}$ in p-type $\mathrm{ZnO}$ DMS will be greater than $300 \mathrm{~K}$, which is very important for making various room temperature electromagnetic devices.

$\mathrm{Mn}$ impurity can be incorporated into $\mathrm{ZnO}$ during its growth by many approaches, including pulsed laser deposition [7], hydrothermal method [8], electron spin resonanc [9], which are complicate technologies. In other researches, $\mathrm{ZnO}$ Ncs are grown using wet chemical methods [10,11], but the wet modality limits the device applications. In our experiment, we try to synthesize and dope $\mathrm{ZnO}$ nanocrystals on $\mathrm{Si}$ and quartz substrates by a simple vapor phase transport (VPT) process [12]. The surface morphology of the products was investigated by

${ }^{1}$ School of Mathematics and Physics, Suzhou University of Science and Technology, 1701 Binhe Road, Suzhou 215011, Jiangsu, China

${ }^{2}$ Nankai University, Tianjin, 300071, China

*Corresponding author. Fax: +86-575-88342415, E-mail: maxy@usx.edu.cn 
scanning electron microscope, and the optical characteristics were analyzed by photoluminescence spectra at roomtemperature. The photoluminescence mechanism for both pure $\mathrm{ZnO}$ nanocrystals and $\mathrm{Mn}$ doped $\mathrm{ZnO}$ are discussed as well.

$\mathrm{ZnO}$ nanocrystals were fabricated by a simple vapor phase transport (VPT) process, as shown in Fig. 1, where the system is consisted of a large horizontal quartz tube furnace, a vacuum system, a gas meter, and a temperature controller. Pure zinc acetate dihydrate as the source material was placed at the bottom of a one-endsealed slender quartz tube that was positioned at the center position of the furnace. For uniform growth, cleaned Si (111) and quartz wafers as the substrates were placed at end of the large tube; in front of the slender tube but far away from it. Prior to the fabrication, the furnace was pumped at vacuum and heated to $100^{\circ} \mathrm{C}$ and kept for 2 hours to remove the water moisture in the zinc acetate dehydrate. Then the furnace was heated to the growing temperature of $500^{\circ} \mathrm{C}$. At the same time, a mixture gas of $\mathrm{O}_{2} / \mathrm{Ar}$ was loaded through the furnace and kept a constant flowing during the growing process. The growing process was carried out for $30 \mathrm{~min}$, and the samples were taken out when the system cooled down to room temperature.

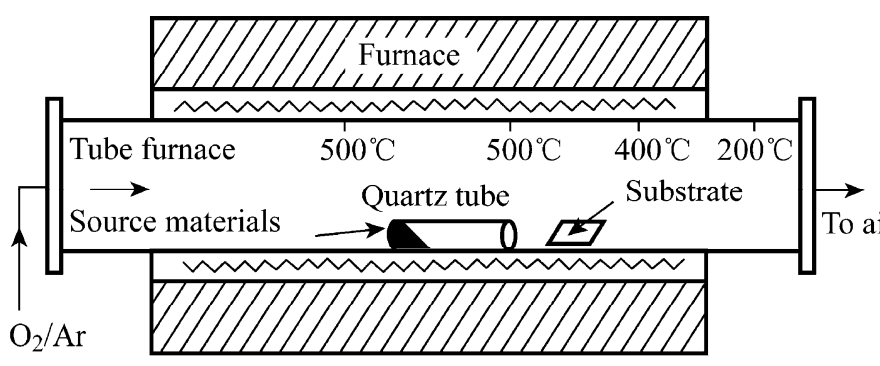

FIG. 1. Schematic diagram of the experimental system.

For $\mathrm{Mn}$ doped $\mathrm{ZnO}$ Ncs, the synthesis process is identical with above except that the source material is the mixture of zinc acetate dihydrate and manganese acetate tetrahydrate with different ratios. After the mixture decomposing at a high temperature of $500{ }^{\circ} \mathrm{C}$, the mixed vapor of $\mathrm{Zn} / \mathrm{Mn}$ was drawn out of the small tube by the flowing gas and condensed on the substrate as well. When the mixed $\mathrm{Zn} / \mathrm{Mn}$ liquid droplets deposited and crystallized, $\mathrm{ZnO}$ Ncs were uniformly doped with Mn.

The morphology of the products was investigated by a Shimadzu SS-550 super scanning electron microscope (SEM). And the composition was analyzed by X-ray diffraction (XRD) on a RINT2000 vertical goniometer with $\mathrm{Cu} \mathrm{Ka}$ radiation $(\lambda=0.1541 \mathrm{~nm})$. The photoluminescence (PL) measurements were performed by using a $310 \mathrm{~nm}$ excitation source at room temperature.

Figure 2(a) illustrates the surface morphology of $\mathrm{ZnO}$ Ncs on Si substrate investigated by SEM. Clearly, $\mathrm{ZnO}$ nanoparticles with an average size of $18 \mathrm{~nm}$ are uniformly dispersed on $\mathrm{Si}$

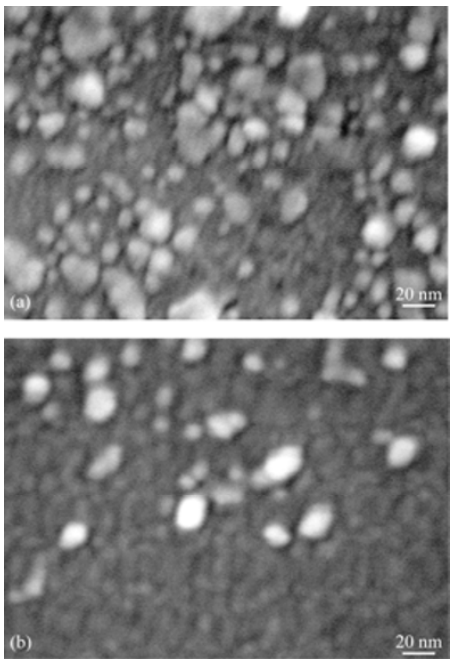

FIG. 2. (a) The surface SEM image of $\mathrm{ZnO}$ Ncs on Si substrate; (b) the surface SEM image of $\mathrm{ZnO}$ Ncs on quartz substrate.

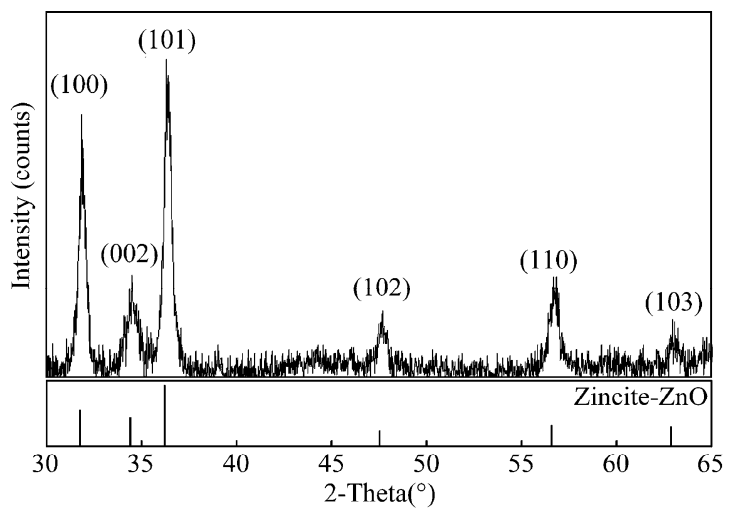

FIG. 3. The X-ray diffraction pattern of $\mathrm{ZnO}$ Ncs on Si substrate. The diffraction peaks corresponds the wurtzite structure of bulk $\mathrm{ZnO}$.

substrate, and a few of them aggregate together, which because new small $\mathrm{ZnO}$ nanoparticles were nucleated continually in the growth process. At the same time, small $\mathrm{ZnO}$ Ncs has large surface effect than that of the bulk material, leading to the nanocrystals readily get together. Figure 2(b) is a SEM picture taken from the quartz sample, where the morphology of $\mathrm{ZnO}$ Ncs is similar to that of Fig. 2(a) except that the density of $\mathrm{ZnO}$ Ncs is lower, indicating that $\mathrm{ZnO}$ Ncs prefer nucleate on $\mathrm{Si}$ substrate to quartz. The X-ray diffraction pattern of the $\mathrm{ZnO}$ Ncs on $\mathrm{Si}$ is shown in the upper panel in Fig. 3, while the standard diffraction spectrum of the bulk wurtzite $\mathrm{ZnO}$ is in the down panel. We can see that the diffraction peaks and the interplane spacing are well matched to the standard diffraction pattern of wurtzite $\mathrm{ZnO}$, demonstrating that our products have a distinct formation of wurtzite $\mathrm{ZnO}$ nanosrystals. The average diameter of $\mathrm{ZnO}$ DQs is $19 \mathrm{~nm}$ estimated from Scherrer's equation. The size is very accordance with the surface SEM image. These results indicate that the $\mathrm{ZnO}$ Ncs obtained in our experiment are of high quality with standard crystal shape and acceptable size. 
Figure 4(a) shows an absorption spectrum of the pure $\mathrm{ZnO}$ Ncs on Si substrate at room temperature. It can be seen that the absorption intensity decreases sharply as the wavelength is over $355 \mathrm{~nm}$, which can be defined as the absorption edge, corresponding the absorption of the intrinsic bandgap of $\mathrm{ZnO}$. From the value of the absorption edge, we can estimate the bandgap of energy of $\mathrm{ZnO} \mathrm{Ncs}$ to be about $3.54 \mathrm{eV}$. Figure 4(b) shows the photoluminescence (PL) spectrum of $\mathrm{ZnO}$ Ncs measured at room temperature. In the PL spectrum, $\mathrm{ZnO}$ Ncs exhibit a strong and predominant UV emission peaked at 377 $\mathrm{nm}$, originating from the band to band emission of $\mathrm{ZnO} \mathrm{Ncs}$. And a much lower blue-green peak is positioned at $435 \mathrm{~nm}$ that is attributed to the surface defect from oxygen vacancies or zinc interstitials. For comparison, the PL spectrum of bulk $\mathrm{ZnO}$ is also given in a red line, where the date is magnified of 100 times. The UV and the blue emissions is absent in the bulk $\mathrm{ZnO}$ at room temperature, while a weak large band peaked at $500 \mathrm{~nm}$ (Kelly color) is appeared, attributing to the emission from the surface energy levels. Therefore, the predominant strong UV emission in $\mathrm{ZnO} \mathrm{Ncs}$ results from the quantum-confined band-edge emission and the quantum size effects. Additionally, In the conventional growth methods, in order to obtain a prominent UV emission, the as grown $\mathrm{ZnO}$ Ncs usually require some accessional treatments, such as surface modification and annealing. In our experiment, $\mathrm{ZnO}$ Ncs sample achieves a very strong UV emission through a simple VPT process without any additional treatment in the experiment, showing that the as grown $\mathrm{ZnO}$ Ncs are of high quality.

Figure 5 shows the absorption spectrum of the Mn doped $\mathrm{ZnO}$ Ncs sample measured at room temperature. There are two

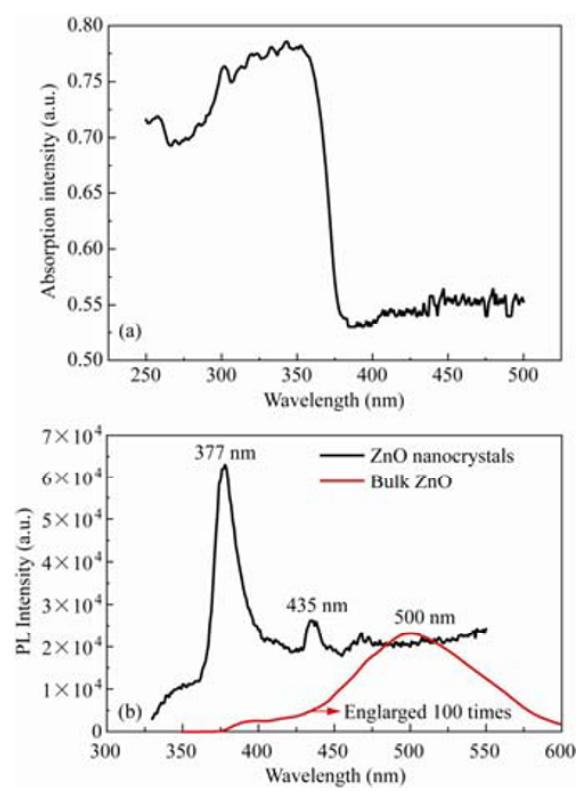

FIG. 4. (a) An absorption spectrum of the pure ZnO Ncs on Si substrate at room temperature; (b) a PL spectra of $\mathrm{ZnO}$ Ncs measured at room temperature, a strong and predominant UV emission peak at $377 \mathrm{~nm}$ originats from the band emission of $\mathrm{ZnO}$ Ncs. For comparison, the PL spectrum of the bulk $\mathrm{ZnO}$ is shown in red line that the datum is enlarged 100 times.

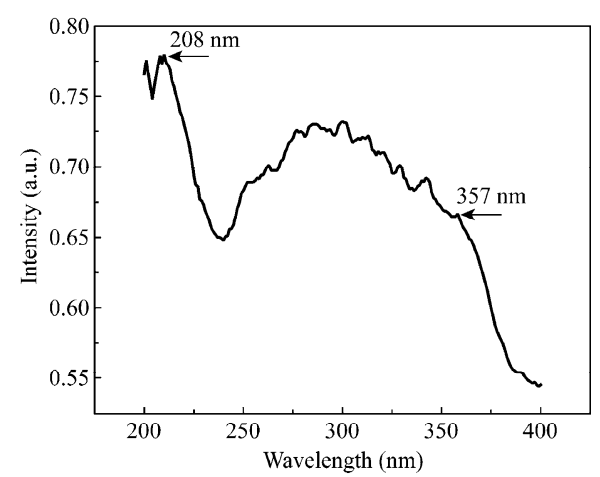

FIG. 5. The absorption spectrum of the Mn doped $\mathrm{ZnO}$ Ncs sample measured at room temperature.

absorption bands, one is located at $208 \mathrm{~nm}$, and the other is a large band with a sharp absorption edge at $357 \mathrm{~nm}$. The former corresponds to the absorption of $\mathrm{MnO}$ that has a larger band gap $(4.2 \mathrm{eV})$, which makes the first absorption edge taking a blue shift. The latter is a combined absorption of ZnO:Mn Ncs, pure $\mathrm{ZnO} \mathrm{Ncs}$, and the surface defect states. Figure 6 shows the photoluminescence spectra of $\mathrm{ZnO}: \mathrm{Mn} \mathrm{Ncs}$ sample. For comparison, the PL spectrum of the pure $\mathrm{ZnO}$ Ncs is shown in panel (a). Figure 6(b) shows the PL spectrum of $\mathrm{ZnO}$ : Mn Ncs when the material ratio of $\mathrm{Zn} / \mathrm{Mn}$ at 95:5. We can see that the PL property has taken a significant change by doping $\mathrm{Mn}$ impurity. The UV emission from the band edge of $\mathrm{ZnO}$ is almost quenching, replaced by two emissions with almost identical intensity in the spectrum, one is located at $409 \mathrm{~nm}$, and the other is placed at $435 \mathrm{~nm}$. The former can be considered as the UV emission shifting to longer wavelength of $408 \mathrm{~nm}$ because of $\mathrm{Mn}$ ions introducing an impurity in the energy

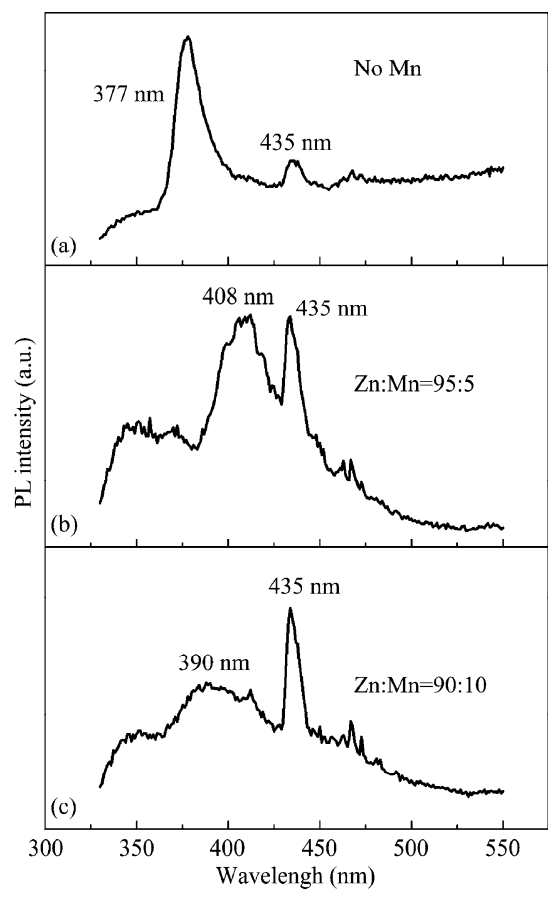

FIG. 6. The PL spectra of ZnO:Mn Ncs grown with different mixing ratio of $\mathrm{Zn} / \mathrm{Mn}$ in source materials; (a) the PL of pure $\mathrm{ZnO}$ Ncs as a control spectrum; (b) the PL of $\mathrm{ZnO}: \mathrm{Mn}$ with the ratio of $\mathrm{Zn} / \mathrm{Mn}$ at 95:5; (c) the PL of $\mathrm{ZnO}: \mathrm{Mn}$ with the ratio of $\mathrm{Zn}: \mathrm{Mn}$ at 90:10. 
bandgap of $\mathrm{ZnO}$. The latter originates from $\mathrm{Mn}$ relevant compounds, such as $\mathrm{Mn}_{3} \mathrm{O}_{4}$ etc. With the ratio of $\mathrm{Zn} / \mathrm{Mn}$ increase to $95: 10$, the blue emission of $435 \mathrm{~nm}$ becomes stronger, while the UV emission decreases much a lot along with shifting from 408 to $390 \mathrm{~nm}$, that is, the major UV emission shifts to short wavelength again.

From the PL spectra, we know that Mn impurity is triumphantly doped into $\mathrm{ZnO}$ Ncs. The doping way of $\mathrm{Mn}$ impurity in $\mathrm{ZnO} \mathrm{Ncs}$ either is an interstitial doping or a substitutional doping. If the substitutional doping occurs, Mn ion will substitute $\mathrm{Zn}$ ion of $\mathrm{ZnO}$ to form $\mathrm{MnO}$. $\mathrm{MnO}$ has a larger band gap $(4.2 \mathrm{eV})$ that may lead to the UV emission taking a blue shift in the PL spectra. However, in our experiment the UV emission shifts to longer wavelength after doping $\mathrm{Mn}$, hence we deduce that the doping is more an interstitial doping than a substitutional doping. In addition, the UV emission shifts to longer wavelength initially, then back to short wavelength again. The similar phenomena also happened in other's researches on $\mathrm{Mn}$ doped $\mathrm{ZnO}[13,14]$, CdS and $\mathrm{ZnSe}$. According to Bylsma's [15] second-order perturbation theory on the similar phenomena in Mn doped $\mathrm{ZnSe}$ bulk materials, we can give a explanation. If the doping concentration of $\mathrm{Mn}$ is low enough $(<2 \%)$, the $d$ orbit of $\mathrm{Mn}$ has strong exchange interactions with the $s$ and $p$ orbits of $\mathrm{ZnO}$, which can be considered as a short range disorder spin system. The first interaction decreases the energy of conduction band bottom, and the second one increases the energy of valence band top, so that the band gap of the product become narrower than before, resulting in the red-shift of UV emission. With the concentration of $\mathrm{Mn}$ increasing $(>2 \%)$, the band gap is broadening, and the emission moves back to the UV field again.

Moreover, the relative intensity ratio of the UV and the blue (435 nm) emissions changes with the ratio of $\mathrm{Zn} / \mathrm{Mn}$ in the source material. With the concentration of Mn increasing, the intensity of the blue emission enhanced much a lot, which comes from surface defect levels associated with oxygen vacancies, $\mathrm{Mn}_{3} \mathrm{O}_{4}$ etc. The enhanced blue emission is mainly attributed to the increasing of Mn impurities on the surface of $\mathrm{ZnO}$ Ncs. Because the decomposing temperature of Mn acetate is higher than that of $\mathrm{Zn}$ acetate, it decomposes later than $\mathrm{Zn}$ acetate in the growth process. Therefore, more Mn impurities are covered on $\mathrm{ZnO}$ Ncs than inter-doped them, on which $\mathrm{Mn}$ further combines with oxygen to form $\mathrm{Mn}_{3} \mathrm{O}_{4}$, a more stable structure, resulting in more oxygen vacancies and enhancing the green emission.

$\mathrm{ZnO}$ Ncs have been grown and doped with Mn by a simple technology of a vapor phase transport (VPT) process. The as grown $\mathrm{ZnO}$ Ncs in size of $19 \mathrm{~nm}$ have a distinct of wurtzite structure. Without any additional treatment, ZnO Ncs sample exhibit a strong and predominant UV emission in the PL spectra at room temperature. For $\mathrm{Mn}$ doped $\mathrm{ZnO} \mathrm{Ncs}$, a UV emission and a blue emission are observed, and the position of the UV emission shifts to longer wavelength direction because of $\mathrm{Mn}$ introduced an impurity level in the bandgap. With the concentration of $\mathrm{Mn}$ increasing, the blue emission enhanced much a lot due to the strong exchange interaction in the short range spin system and the excess $\mathrm{Mn}_{3} \mathrm{O}_{4}$ on the surface, respectively.

This work was supported in parts by the National Natural Science Foundation of China (No. 60776004, 60976071) and the Laboratory for Thin Film Microfabrication of the Ministry of Education.

Received 3 December 2009; accepted 15 December 2009; published online 20 December 2009.

\section{References}

1. X. D. Gao, X. M. Li and W. D. Yu, Mater. Res. Bull. 40, 1104 (2005). doi:10.1016/j.materresbull. 2005.03.018

2. H. S. Kang, B. D. Ahn, J. H. Kim, G. H. Kim, S. H. Lim, H. W. Chang and S. Y. Lee, Appl. Phys. Lett. 88, 202108 (2006). doi:10.1063/1.2203952

3. G. D. Yuan, Z. Z. Ye, L. P. Zhu, Q. Qian, B. H. Zhao, R. X. Fan, Craig L. Perkins and S. B. Zhang, Appl. Phys. Lett. 86, 202106 (2005). doi:10.1063/1.1928318

4. S. K. Mandal and T. K. Nath, Thin solid films 515, 2535 (2006). doi:10.1016/j.tsf.2006.03.032

5. T. Dietl, H. Ohno, F. Matsukura, J. Cibert and D. Ferrand, Science 287, 1019 (2000). doi:10.1126/sci ence.287. 5455.1019

6. T. Dietl, Semicond. Sci. Technol. 17, 377 (2002). doi:10.1088/0268-1242/17/4/310

7. E. De. Posada, G. Tobin, E. McGlynn and J. G. Lunney, Appl. Surf. Sci. 208, 589 (2003).

8. Y. Polyakov, N. B. Smirnov, A. V. Govorkov, E. A. Kozhukhova, Y. W. Heo, M. P. Ivill, K. Ip, D. P. Norton, S. J. Pearton, J. Kelly, R. Rairigh, A. F. Hebard and T. Steiner, J. Vac. Sci. Technol. B 23, 1 (2005).

9. M. Berciu and R. N. Bhatt, Phys. Rev. Lett. 87, 107203 (2001).

10. H. Zhou, H. Alves and D. M. Hofmann, Appl. Phys. Lett. 80, 210 (2002). doi:10.1063/1.1432763

11. J. H. Li and J. Y. Zhang, Chin. J. Liquid Cryst. Display 21, 615 (2006).

12. J. G. Lu, Z. Z. Ye and J. Y. Huang, Appl. Phys. Lett. 88, 063110 (2006). doi:10.1063/1.2172154

13. R. Viswanatha, S. Sapra and S. S. Gupta, J. Phys. Chem. B 108, 6303 (2004). doi:10.1021/jp049960o

14. X. T. Zhang, Y. C. Liu and J. Y. Zhang, J. Cryst. Growth 243, 80 (2003). doi:10.1016/S0022-0248(03)01143-6

15. R. B. Bylsma, W. M. Becker and J. Kossut, Phys. Rev. B 33, 8207 (1986). doi:10.1103/PhysRevB.33.8207 